IHES/P/94/22

CPT-94/P.E.3021

\title{
Orbital tests of relativistic gravity using artificial satellites
}

\author{
Thibault Damour \\ Institut des Hautes Etudes Scientifiques, 91440 Bures sur Yvette, France \\ and Département d'Astrophysique Relativiste et de Cosmologie, Observatoire de Paris, \\ Centre National de la Recherche Scientifique, 92195 Meudon, France \\ Gilles Esposito-Farèse \\ Centre de Physique Théorique, Centre National de la Recherche Scientifique, \\ Luminy, Case 907, 13288 Marseille Cedex 9, France
}

(March 28, 1994)

\begin{abstract}
We reexamine non-Einsteinian effects observable in the orbital motion of low-orbit artificial Earth satellites. The motivations for doing so are twofold: (i) recent theoretical studies suggest that the correct theory of gravity might contain a scalar contribution which has been reduced to a small value by the effect of the cosmological expansion; (ii) presently developed space technologies should soon give access to a new generation of satellites endowed with drag-free systems and tracked in three dimensions at the centimeter level. Our analysis suggests that such data could measure two independent combinations of the Eddington parameters $\bar{\beta} \equiv \beta-1$ and $\bar{\gamma} \equiv \gamma-1$ at the $10^{-4}$ level and probe the time variability of Newton's "constant" at the $\dot{G} / G \sim 10^{-13} \mathrm{yr}^{-1}$ level. These tests would provide well-needed complements to the results of the Lunar Laser Ranging experiment, and of the presently planned experiments aiming at measuring $\bar{\gamma}$. In view of the strong demands they make on the level of non-gravitational perturbations, these tests might require a dedicated mission consisting of an optimized passive drag-free satellite.
\end{abstract}

PACS number(s): 04.80.Cc, 04.25.Nx, 95.40.+s 


\section{INTRODUCTION}

Though relativistic gravity has already been probed by quite a few experimental tests, with the conclusion that General Relativity is in agreement with all existing experiments (see [1,2] for reviews), recent theoretical developments in tensor-scalar cosmological models suggest that the correct theory of gravity might differ from Einstein's theory by containing a scalar contribution whose present magnitude has been naturally driven to a small value by the cosmological expansion [3, 1]. These theoretical results provide a new motivation for trying to improve the existing tests of tensor-scalar theories of gravity.

In this paper we reexamine the use of artificial (Earth) satellites to perform orbital tests of non-Einsteinian gravity. We shall consider here only the most conservative deviations from Einstein theory, i.e. boost-invariant effects associated to the exchange of scalar excitations. [We refer to our recent work [5] for a discussion of the use of satellite data as probes of possible preferred-frame effects in relativistic gravity.] Although several of the Einsteinian or non-Einsteinian effects in the motion of artificial satellites have been abundantly discussed in the literature [1,6], it seems that there exists no treatment of the problem which is both systematic and observation-oriented. There exists a systematic analysis of non-Einsteinian effects in the motion of the Moon [9], but we shall see that the two problems are quite dissimilar both because of differences in physical parameters $\left(a_{\text {Moon }} / R_{\text {Earth }} \sim 60\right.$ compared to $a_{\text {satellite }} / R_{\text {Earth }} \sim$ a few) and of differences in observational techniques (radial ranging for the Moon versus three-dimensional tracking for satellites). The aim of the present paper is to provide a systematic analysis of non-Einsteinian effects possibly observable in the orbital data of drag-free satellites whose three-dimensional motion is tracked (either by Laser or by Global Positioning System (GPS) techniques) at the centimeter level. The Gravity Probe B (GPB) satellite should soon provide the first example of such satellites. [The existing Laser Geodynamical Satellites (LAGEOS) are tracked with centimeter precision, but the small level of residual drag acting on their motion is probably too large for them to be useful probes of the effects we discuss below.]

Note that, contrary to some proposals, e.g. [7], we shall not consider explicitly here the purely Einsteinian relativistic effects. We consider that the ensemble of existing positive tests of General Relativity [1,2] has already probed most of the qualitatively crucial features of Einstein's theory. We shall therefore assume that the Einsteinian post-Newtonian contributions to the satellite equations of motion are separately included in their entirety (see [10]), and focus on the quantitatively most promising effects for measuring possible non-Einsteinian weak-field deviations.

In the post-Newtonian limit, generic tensor-scalar theories of gravity predict that the motion of $N$ spherical, non rotating bodies is given by the Lagrangian [11]

$$
\begin{aligned}
L_{\bar{\beta}, \bar{\gamma}, \bar{\delta}}= & -\sum_{A} m_{A} c^{2}\left(1-\mathbf{v}_{A}^{2} / c^{2}\right)^{1 / 2} \\
& +\frac{1}{2} \sum_{A \neq B} \frac{G_{A B} m_{A} m_{B}}{r_{A B}}\left[1+\frac{3}{2 c^{2}}\left(\mathbf{v}_{A}^{2}+\mathbf{v}_{B}^{2}\right)\right. \\
& -\frac{7}{2 c^{2}}\left(\mathbf{v}_{A} \cdot \mathbf{v}_{B}\right)-\frac{1}{2 c^{2}}\left(\mathbf{n}_{A B} \cdot \mathbf{v}_{A}\right)\left(\mathbf{n}_{A B} \cdot \mathbf{v}_{B}\right)
\end{aligned}
$$




$$
\begin{gathered}
\left.+\frac{\bar{\gamma}}{c^{2}}\left(\mathbf{v}_{A}-\mathbf{v}_{B}\right)^{2}\right] \\
-\frac{1}{2} \sum_{B \neq A \neq C}(1+2 \bar{\beta}) \frac{G^{2} m_{A} m_{B} m_{C}}{c^{2} r_{A B} r_{A C}}
\end{gathered}
$$

where $r_{A B} \equiv\left|\mathbf{x}_{A}-\mathbf{x}_{B}\right|, \mathbf{n}_{A B} \equiv\left(\mathbf{x}_{A}-\mathbf{x}_{B}\right) / r_{A B}, \mathbf{v}_{A} \equiv d \mathbf{x}_{A} / d t$, and where the summation symbols denote multiple sums over the various independent body labels $A, B, \ldots$ with the only exclusion of the would be infinite contributions.

In Eq. (1.1) the effective coupling constant $G_{A B}$ for the gravitational interaction of bodies $A$ and $B$ is of the form

$$
G_{A B}=G\left[1+\bar{\delta}_{A}+\bar{\delta}_{B}\right]
$$

where $\bar{\delta}_{A}$ is generically the sum of two physically independent contributions

$$
\bar{\delta}_{A}=\widehat{\delta}_{A}+\eta \frac{E_{A}^{\mathrm{grav}}}{m_{A} c^{2}},
$$

with

$$
\eta \equiv 4 \bar{\beta}-\bar{\gamma}
$$

The first contribution $\widehat{\delta}_{A}$ comes from the fact that the physically best motivated tensorscalar theories violate the weak equivalence principle [4], while the contribution proportional to the gravitational self-energy

$$
E_{A}^{\text {grav }}=-(G / 2) \int_{A} \int_{A} d^{3} x d^{3} x^{\prime} \rho(\mathbf{x}) \rho\left(\mathbf{x}^{\prime}\right) /\left|\mathbf{x}-\mathbf{x}^{\prime}\right|
$$

derives from the fact that all the theories containing a variable local gravitational "constant" violate the strong equivalence principle [12].

The dimensionless numbers $\bar{\beta}, \bar{\gamma}$ parametrize weak-field deviations from General Relativity and are proportional to the strength (relative to the usual tensor contribution) of the coupling to matter of the scalar contribution to gravity [11]. They are related to the usual post-Newtonian (Eddington) parameters simply by $\bar{\beta} \equiv \beta-1, \bar{\gamma} \equiv \gamma-1$ (so that $\eta=4 \beta-\gamma-3$ ). Besides the effects parametrized by $\bar{\beta}, \bar{\gamma}$ and the $\bar{\delta}$ 's, another deviation from General Relativity generically predicted by tensor-scalar theories, and implicitly contained in the Lagrangian (1.1), is a possible time variability of the gravitational coupling strength $G$, as well as (in generic tensor-scalar theories [4]) a time variability of the masses $2 m_{A}$.

\footnotetext{
${ }^{1}$ In Eq. (1.2), the basic gravitational constant $G$ is the sum of the contribution due to the tensor interaction, and of a mean contribution due to the scalar interaction. One assumes that the small composition-dependent effects $\bar{\delta}_{A} \ll 1$, and neglects terms of order $\bar{\delta}^{2}$.

${ }^{2}$ The other parameters $\bar{\beta}, \bar{\gamma}, \bar{\delta}_{A}$ are generically also slowly changing. However, this leads to higher-order, unobservably small effects.
} 
The best present (one sigma) observational limits on the parameters of the Lagrangian (1.1) measuring deviations from Einstein's theory are: $|\bar{\gamma}|<2 \times 10^{-3}$ [13], $|\bar{\beta}|<3 \times 10^{-3}$ (assuming a Sun's $\left.J_{2} \sim 2 \times 10^{-7}\right)$ [14], $\bar{\delta}_{\text {Moon }}-\bar{\delta}_{\text {Earth }}=(-2.7 \pm 6.2) \times 10^{-13}$ [15], and $|\dot{G} / G| \lesssim 10^{-11} \mathrm{yr}^{-1} \| 14$ (we are using the fact that combined geophysical and astronomical data set a bound of order $10^{-13} \mathrm{yr}^{-1}$ on the possible time variability of the masses [16]). Note that if one were to assume the exact validity of the weak equivalence principle, i.e. $\widehat{\delta}_{A} \equiv 0$, the Lunar Laser Ranging result on $\bar{\delta}_{\text {Moon }}-\bar{\delta}_{\text {Earth }}$ would imply $\eta=(-0.6 \pm 1.4) \times 10^{-3}$, from which - using the Viking limit - one would deduce $\bar{\beta}=(-1 \pm 5) \times 10^{-4}$ [15]. However, in the present work we have in mind the general class of tensor-scalar theories which do not respect the weak equivalence principle (such as in Ref. 四). As the present observational limits on the $\widehat{\delta}_{A}$ 's are not better than $3 \times 10^{-12}$ [17], we must consider the $\bar{\delta}_{A}$ 's in Eq. (1.1) as being independent from $\bar{\beta}$ and $\bar{\gamma}$.

The object of the present paper is to point out that the centimeter-level tracking of drag-free artificial Earth satellites is a promising tool for measuring both $\bar{\beta}$ and $\bar{\gamma}$ at the $10^{-4}$ level, and $\dot{G} / G$ at the $10^{-13} \mathrm{yr}^{-1}$ level. We shall show that there are two independent relativistic effects which build up over many orbital periods and lead to displacements $O(\bar{\beta}, \bar{\gamma}) \times 10^{4} \mathrm{~cm}$ for integration times of the order of 6 months. One of these two relativistic effects is non null and is the well known perigee advance associated with the (Eddingtonmodified) Schwarzschild field of the Earth which has been abundantly discussed in the literature [1,6,8, 18]. The other one is a null relativistic effect whose origin is known [1,9], but whose importance, in the context of low orbit satellites, for testing the combination $\eta=4 \bar{\beta}-\bar{\gamma}$ has (as far as we are aware) not been clearly realized before. [See further discussion below.]

\section{LAGRANGIAN AND EQUATIONS FOR MOTION FOR SATELLITE MOTION.}

The Lagrangian (1.1) describes the global dynamics of the bodies of the solar system ("barycentric" reference frame). We wish to pass to a "geocentric" reference frame. A rigorous and elegant way of effecting such a transformation in the framework of General Relativity has been recently discussed in Ref. [10]. However, we are here working within a non-general-relativistic framework. The safest way of deriving the deviations from a general relativistic description of the geocentric motion of a satellite is to start from the $\bar{\beta}-\bar{\gamma}-\bar{\delta}$ modified barycentric equations of motion of the Earth and the satellite and to take their difference. We restrict our attention to the effects associated with the satellite (label 1), the Earth (label 2) and the Sun (label 3), and introduce the notation[ $\mathbf{r} \equiv r \mathbf{n} \equiv \mathbf{x}_{12} \equiv \mathbf{x}_{1}-\mathbf{x}_{2}$, $\mathbf{v} \equiv \dot{\mathbf{r}}, D \mathbf{N} \equiv \mathbf{x}_{32} \equiv \mathbf{x}_{3}-\mathbf{x}_{2}, \mathbf{V} \equiv \dot{\mathbf{x}}_{32} \equiv \mathbf{v}_{3}-\mathbf{v}_{2}, m \equiv m_{2}$ and $M \equiv m_{3}$

The general relativistic equations of motion exhibit some remarkable features called "effacement properties" in Ref. [19]. There is an effacement of the internal structures of the gravitating bodies in the sense that, to a high accuracy, one can write the equations of

\footnotetext{
${ }^{3}$ We do not need a special notation for the mass of the satellite as it disappears from the final equations.
} 
motion in terms of only some "centers of mass" and some "masses". There is also an effacement of the external universe in the dynamics of a local gravitating system. For instance, after introducing some special geocentric coordinate frame, the influence of the Sun on the dynamics of an Earth satellite is effaced up to terms which decrease like $D^{-3}$ when $D \rightarrow \infty$. Both types of effacement properties are violated in tensor-scalar theories. The violation of the first type of effacement properties shows up clearly in the Lagrangian (1.1) through the composition dependence of the effective coupling constant $G_{A B}$. The violation of the second type of effacement properties shows up in the fact that the geocentric equations of motion of a satellite will contain terms proportional to $G M / c^{2} D$ and $G M / c^{2} D^{2}$ (with coefficients linear in $\bar{\beta}, \bar{\gamma}$ and $\bar{\delta}$ ) which cannot be eliminated by a coordinate transformation. In this paper, we shall be especially interested in the leading $\left(\propto G M / c^{2} D\right)$ and subleading $\left(\propto G M / c^{2} D^{2}\right)$ violations of the effacement of external influences appearing in non-Einsteinian theories. We shall neglect the terms containing a factor $G M / c^{2} D^{3}$ which are, roughly speaking, nonEinsteinian relativistic corrections to the Newtonian tidal forces and are therefore extremely small'f.

It is convenient to simplify the geocentric equations of motion by rescaling the local spatial coordinates according to

$$
\mathbf{r}^{\text {new }}=\left(1+\bar{\gamma} \frac{G M}{c^{2} D}\right) \mathbf{r}^{\text {old }}
$$

After this rescaling, the geometric equations of motion have the form

$$
\ddot{\mathbf{r}}=\mathbf{A}_{\mathrm{GR}}+\mathbf{B}
$$

with

$$
\begin{aligned}
\mathbf{A}_{\mathrm{GR}}=-\frac{G m}{r^{2}} \mathbf{n} & -\frac{G M}{D^{3}}[\mathbf{r}-3(\mathbf{r} \cdot \mathbf{N}) \mathbf{N}] \\
& +O\left(\frac{G M}{D^{4}}\right)+O\left(\frac{1}{c^{2}}\right),
\end{aligned}
$$

denoting the general relativistic acceleration term (see [10 for the full expression of $\mathbf{A}_{\mathrm{GR}}$ including a relativistic treatment of tidal forces), and

$$
\mathbf{B}=\mathbf{B}_{0}+\mathbf{B}_{1}+\mathbf{B}_{2}+O\left[\frac{G M}{D^{3}}\left(\frac{\bar{\beta}}{c^{2}}+\frac{\bar{\gamma}}{c^{2}}+\bar{\delta}\right)\right]
$$

denoting the expansion of the non-Einsteinian acceleration terms in successive powers of $D^{-1}$ :

\footnotetext{
${ }^{4}$ Compared to the leading Newtonian acceleration, they are typically smaller by a factor $\lesssim$ $\bar{\gamma}(V / c)^{2} M r^{3} / m D^{3} \lesssim 5 \times 10^{-19}\left(a / R_{\text {Earth }}\right)^{3}$.

${ }^{5}$ This rescaling is the $\bar{\gamma}$-dependent part of a standard rescaling in the PPN literature [1],6]. Its effect is to efface the term proportional to $\bar{\gamma} G M / c^{2} D$ in the spatial metric $g_{i j}$.
} 


$$
\begin{aligned}
\mathbf{B}_{0}= & \frac{G m}{r^{2}} \mathbf{n}\left[2(\bar{\beta}+\bar{\gamma}) \frac{G m}{c^{2} r}-\bar{\gamma} \frac{v^{2}}{c^{2}}-\bar{\delta}_{1}-\bar{\delta}_{2}\right] \\
& +\frac{2 \bar{\gamma}}{c^{2}} \frac{G m}{r^{2}}(\mathbf{n} \cdot \mathbf{v}) \mathbf{v} \\
\mathbf{B}_{1}= & (4 \bar{\beta}-\bar{\gamma}) \frac{G m}{c^{2} D} \frac{G m}{r^{2}} \mathbf{n}, \\
\mathbf{B}_{2}= & \frac{G M}{D^{2}} \mathbf{N}\left[-2 \bar{\beta} \frac{G m}{c^{2} r}+\bar{\delta}_{1}-\bar{\delta}_{2}\right] \\
& +2(\bar{\beta}+\bar{\gamma}) \frac{G M}{D^{2}} \frac{G m}{c^{2} r}(\mathbf{n} \cdot \mathbf{N}) \mathbf{n} \\
& +\frac{\bar{\gamma}}{c^{2}} \frac{G M}{D^{2}}\left[\mathbf{v}^{2} \mathbf{N}-2(\mathbf{v} \cdot \mathbf{N}) \mathbf{v}\right] \\
& +2 \frac{\bar{\gamma}}{c^{2}} \frac{G M}{D^{2}}(\mathbf{N} \times \mathbf{V}) \times \mathbf{v} .
\end{aligned}
$$

To illustrate the non-Einsteinian terms proportional to $G M / D^{3}$ we can quote its numerically dominant contribution:

$$
\mathbf{B}_{3}=-\left(\bar{\delta}_{1}+\bar{\delta}_{3}\right) \frac{G M}{D^{3}}[\mathbf{r}-3(\mathbf{r} \cdot \mathbf{N}) \mathbf{N}]+\cdots
$$

Let us also note that we shall neglect in this work the non-Einsteinian acceleration terms depending on the higher (mass and spin) multipole moments of the Earth. [We assume however that the Einsteinian effects of these is fully taken into account in $\mathbf{A}_{\mathrm{GR}}$.] For instance, the spin-orbit interaction adds a term of the form $\mathbf{B}_{S}=\mathbf{v} \times \mathbf{H}_{\bar{\gamma}}$ to $\mathbf{B}$. Here $\mathbf{H}_{\bar{\gamma}}=-\frac{\bar{\gamma}}{c^{2}} \boldsymbol{\nabla} \times$ $\left(G \mathbf{S}_{2} \times \mathbf{r} / r^{3}\right)$ where $\mathbf{S}_{2}$ is the spin angular momentum of the Earth, so that

$$
\mathbf{B}_{S}=\bar{\gamma} \frac{G}{c^{2} r^{3}}\left[\mathbf{v} \times \mathbf{S}_{2}-3\left(\mathbf{n} \cdot \mathbf{S}_{2}\right) \mathbf{v} \times \mathbf{n}\right] .
$$

The spin-orbit acceleration $\mathbf{B}_{S}$ is smaller than the velocity-dependent terms in $\mathbf{B}_{0}$, Eq. (2.5), by a factor $S_{2} /(m r v) \sim 0.02\left(a / R_{\text {Earth }}\right)^{-1 / 2}$. The other multipole contributions will contain even smaller factors. They can be therefore safely neglected with respect to the leading $\bar{\gamma}$ and $\bar{\beta}$-dependent terms kept in Eqs. (2.5)-(2.7).

The last term on the right-hand side of Eq. (2.7) has the form of a Coriolis force, $2 \Omega_{\bar{\gamma}} \times \mathbf{v}$, with

$$
\Omega_{\bar{\gamma}}=\bar{\gamma} \frac{G M}{c^{2} D^{2}} \mathbf{N} \times \mathbf{V}
$$

As first discovered by De Sitter a similar relativistic Coriolis term, $2 \boldsymbol{\Omega}_{\mathrm{GR}} \times \mathbf{v}$, with

$$
\mathbf{\Omega}_{\mathrm{GR}} \simeq \frac{3}{2} \frac{G M}{c^{2} D^{2}} \mathbf{N} \times \mathbf{V}
$$

is present in Einstein's theory if one works in a geocentric frame which has a fixed orientation with respect to the barycentric frame. Alternatively, one can efface away the total relativistic Coriolis force from the local equations of motion by working in a geocentric frame which rotates with angular velocity 


$$
\Omega_{\mathrm{tot}} \equiv \Omega_{\mathrm{GR}}+\Omega_{\bar{\gamma}} \simeq\left(1+\frac{2}{3} \bar{\gamma}\right) \Omega_{\mathrm{GR}}
$$

with respect to the barycentric frame. [See [10] for a more accurate treatment of $\Omega_{\mathrm{GR}}$ than the one given here.] Let us note that the rotation $\Omega_{\text {tot }}$ ("precession of the local Earth inertial frame") is acting upon all the satellite orbits (including the Moon), as well as upon the solid Earth. Therefore $\Omega_{\text {tot }}$ essentially disappears in the position measurements effected by means of GPS or Laser techniques. It appears however when connecting local measurements to global ones, e.g. through the use of Very Large Baseline Interferometry (VLBI) techniques. It appears also in the Lunar Laser Ranging (LLR) data because the internal dynamics of the Earth-Moon system is strongly influenced by the Sun. The measurement of $\boldsymbol{\Omega}_{\mathrm{tot}}$ through LLR data is now reaching the $1 \%$ level (more precisely $(2 / 3) \bar{\gamma}=-0.3 \pm 0.9 \%$ [15]). As the effects of $\Omega_{\text {tot }}$ on low satellite orbits are more difficult to observe this does not look as a promising way of getting improved tests of $\bar{\gamma}$. Therefore, in the following, we shall concentrate on the other non-Einsteinian effects B in Eq. (2.2). In other words, we shall study the satellite motion in a geocentric frame precessing with the velocity (2.12), so that the last term in Eq. (2.7) is absent.

In such a dynamically non rotating geocentric frame one can verify that the equations of motion of a satellite derive from a Lagrangian of the form

$$
\begin{aligned}
& L=L_{\mathrm{GR}}+R \\
& L_{\mathrm{GR}}=\frac{1}{2} \mathbf{v}^{2}+\frac{G m}{r}-\frac{G M}{2 D^{3}}\left[\mathbf{r}^{2}-3(\mathbf{N} \cdot \mathbf{r})^{2}\right] \\
& + \text { other Newtonian and post-Newtonian contributions, } \\
& R=R_{0}+R_{1}+R_{2}+O\left[\frac{G M}{D^{3}}\left(\frac{\bar{\beta}}{c^{2}}+\frac{\bar{\gamma}}{c^{2}}+\bar{\delta}\right)\right] \\
& R_{0}=\left[\bar{\delta}_{1}+\bar{\delta}_{2}+\bar{\gamma} \frac{\mathbf{v}^{2}}{c^{2}}-\bar{\beta}_{\frac{G m}{c^{2} r}}\right] \frac{G m}{r} \\
& R_{1}=-(4 \bar{\beta}-\bar{\gamma}) \frac{G M}{c^{2} D} \frac{G m}{r}, \\
& R_{2}=\left(\bar{\gamma} \frac{\mathbf{v}^{2}}{c^{2}}-2 \bar{\beta} \frac{G m}{c^{2} r}+\bar{\delta}_{1}-\bar{\delta}_{2}\right) \frac{G M}{D^{2}}(\mathbf{N} \cdot \mathbf{r}) .
\end{aligned}
$$

\footnotetext{
${ }^{6}$ They correspond to spatial displacements $\Omega_{\mathrm{tot}} a=\left(1+\frac{2}{3} \bar{\gamma}\right)\left(a / R_{\text {Earth }}\right) \times 59 \mathrm{~cm} \mathrm{yr}^{-1}$.
} 


\section{NON-EINSTEINIAN EFFECTS IN THE MOTION OF SATELLITES}

As we are discussing small deviations from a general relativistic motion, we can discuss independently the perturbations depending upon different powers of $D^{-1}$ in Eq. (2.15) and superpose linearly their effects.

The terms which are independent from the presence of the Sun, Eqs. (2.5) and (2.16), are well known. Apart from a constant renormalization of $G \rightarrow G_{\text {effective }} \equiv G\left[1+\bar{\delta}_{1}+\bar{\delta}_{2}\right]$ (whose possible dependence upon the satellite's composition is too small to be of observational significance), they correspond to a geodesic motion in an Eddington-modified Schwarzschild metric. The corresponding solution can be written down explicitly (see e.g. [20] and Appendix A.2.3 of [6] for a simple form of the explicit solution at the post-Newtonian accuracy). Short-period perturbations correspond to spatial displacements of order $(\bar{\beta}+\bar{\gamma}) \mathrm{Gm} / \mathrm{c}^{2}$, i.e. smaller than about $10^{-3} \mathrm{~cm}$ given the existing limits on $\bar{\beta}$ or $\bar{\gamma}$. This is too small to be of observational significance. Finally, the only observationally significant effect coming from $\mathbf{B}_{0}$ is the secular advance of the perigee

$$
\delta_{0} \omega=(2 \bar{\gamma}-\bar{\beta}) \frac{G m}{c^{2} a\left(1-e^{2}\right)} n t
$$

where $a$ denotes the semi-major axis of the orbit, $e$ its eccentricity and $n \equiv 2 \pi / P \simeq$ $\left(G m / a^{3}\right)^{1 / 2}$ the orbital frequency [The index zero on the left-hand side of (3.1) refers to the cause $\mathbf{B}_{0}$ of this effect]. In terms of the ratio $\widehat{a} \equiv a / R$ where $R=6.371 \times 10^{8} \mathrm{~cm}$ is the Earth radius, one finds]

$$
a \delta_{0} \omega \simeq(2 \bar{\gamma}-\bar{\beta}) \frac{\widehat{a}^{-3 / 2}}{1-e^{2}} \times\left(\frac{t}{1 \mathrm{yr}}\right) \times 1.74 \times 10^{4} \mathrm{~cm}
$$

The terms which are proportional to $G M / D$, Eqs. (2.6) and (2.17) are, in principle, well known. They can be simply interpreted as associated with a renormalization of the local (geocentric) value of $G$ due to the proximity of the Sun: $G \rightarrow G_{\text {loc }}=\left(1-\eta G M / c^{2} D\right) G$ [1]. The existence of this (time-dependent) renormalization was taken into account in the treatment of non-Einsteinian effects in Lunar Laser Ranging [9] but was found to lead to a very small perturbation of the (directly observed) radial distance (about 140 times smaller than the effects associated with the violation of the equivalence principle, $\bar{\delta}_{1}-\bar{\delta}_{2} \neq 0$ in Eq. (2.7)). This conclusion is correct in the case of the Moon, but the main point of the present paper is to emphasize that the situation is very different in the case of low Earth satellites for two different reasons: (a) their proximity to the Earth enhances the perturbation, and (b) the fact that their motion can be tracked in three dimensions gives access to the longitudinal displacement which happens to be enhanced by a large factor $\propto n / n_{2}$, where $n_{2} \equiv\left(G M / a_{2}^{3}\right)^{1 / 2}$ denotes the orbital frequency of the Earth around the Sun.

\footnotetext{
${ }^{7}$ Numerically $P=\widehat{a}^{3 / 2} \times 1.406 \mathrm{~h}, G m / c^{2}=0.4435 \mathrm{~cm}, G m / R c^{2}=6.96 \times 10^{-10}$.
} 
It is possible to solve for the effects of $R_{1}$, Eq. (2.17), by the following method. Let us introduce new space and time variables 8

$$
\begin{aligned}
& \mathbf{r}^{\prime}=\mathbf{r}-\varepsilon=\left(1-\eta \frac{G M}{c^{2} D}\right) \mathbf{r} \\
& t^{\prime}=t-\varepsilon^{0}=t-2 \eta \int d t \frac{G M}{c^{2} D}
\end{aligned}
$$

The difference between the Lagrangian $L^{\prime}$ describing the motion in the new variables and the original Lagrangian $L$ is given by 21]

$$
L^{\prime}-L=\frac{\delta L}{\delta \mathbf{r}}\left(\varepsilon-\varepsilon^{0} \mathbf{v}\right)+\frac{d}{d t} Q(\varepsilon)
$$

where $\delta L / \delta \mathbf{r}=-\left(\ddot{\mathbf{r}}+G m \mathbf{n} / r^{2}\right)+O\left(G M / D^{3}\right)+O\left(1 / c^{2}\right)$ and where $d Q / d t$ is some total time derivative. Using the identities

$$
\begin{aligned}
\mathbf{r} \cdot\left(\ddot{\mathbf{r}}+G m \frac{\mathbf{n}}{r^{2}}\right) & \equiv-\mathbf{v}^{2}+\frac{G m}{r}+\frac{1}{2} \frac{d^{2} \mathbf{r}^{2}}{d t^{2}}, \\
\mathbf{v} \cdot\left(\ddot{\mathbf{r}}+G m \frac{\mathbf{n}}{r^{2}}\right) & \equiv \frac{d}{d t}\left(\frac{1}{2} \mathbf{v}^{2}-\frac{G m}{r}\right),
\end{aligned}
$$

and operating by parts one finds

$$
\begin{aligned}
L^{\prime}-L=\eta \frac{G M}{c^{2} D} \frac{G m}{r} & -\frac{1}{2} \eta \mathbf{r}^{2} \frac{d^{2}}{d t^{2}}\left(\frac{G M}{c^{2} D}\right)+\frac{d}{d t} Q^{\prime}(\varepsilon) \\
& +O\left(\frac{G M}{D^{3}} \varepsilon\right)+O\left(\frac{\varepsilon}{c^{2}}\right) .
\end{aligned}
$$

The first term on the right-hand side of (3.6) cancels the contribution $R_{1}$ to $L$, while the second term is easily seen to be of order $\eta(V / c)^{2} G M r^{2} / D^{3}$, i.e. of order of the non-Einsteinian relativistic corrections to tidal effects ( see $\mathbf{B}_{3}$, Eq. (2.8)) that we are neglecting in this work.

The conclusion is that the effect of $R_{1}$ is equivalent to performing the transformation (3.3) on the solution $\mathbf{r}^{\prime}\left(t^{\prime}\right)$ of the equations of motion in which $R_{1}$ (and $\mathbf{B}_{1}$ ) have been set equal to zero. Note that this method can be applied either to the full term $G M / c^{2} D$ or only to its time-varying piece due to the eccentricity $e_{2}$ of the Earth orbit around the Sun (we neglect $\left(e_{2}\right)^{2}$ for simplicity):

$$
\frac{\widetilde{G M}}{c^{2} D} \equiv \frac{G M}{c^{2} D}-\frac{G M}{c^{2} a_{2}} \simeq e_{2} \frac{G M}{c^{2} a_{2}} \cos n_{2}\left(t-t_{2}\right),
$$

where $t_{2}$ is the time of passage of the Earth at its perihelion. The time-independent renormalization of $G$ associated with $G M / c^{2} a_{2}$ is locally unobservable. The time-dependent

\footnotetext{
${ }^{8}$ We do not consider $\mathbf{r}^{\prime}$ and $t^{\prime}$ as new coordinates in space-time but only as auxiliary variables which are helpful to solve the equations of motion. Note that $t^{\prime}$ would have bad properties as a time coordinate as it would introduce a term linear in $\eta G M / c^{2} D$ in the metric component $g_{00}^{\prime}$.
} 
perturbations in the spatial positions, Eq. (3.3a), are of order $0.1 \eta \hat{a} \mathrm{~cm}$, i.e. too small to be observable. Finally, the only observationally significant effect coming from $\mathbf{B}_{1}$ is the change, given by Eq. (3.3b), in the time according to which the satellite runs on its orbit. If we introduce the mean anomaly $\ell$, i.e. an angle connected with the position on the orbit which, in the unperturbed motion, varies linearly in time, we shall have in the perturbed motion

$$
\ell=n t^{\prime}+\text { const }=n\left(t-2 \eta \int d t \frac{\widetilde{G M}}{c^{2} D}\right)+\text { const }
$$

or $\ell=n t+\delta_{1} \ell$ with

$$
\delta_{1} \ell=-2 \eta e_{2} \frac{G M}{c^{2} a_{2}} \frac{n}{n_{2}} \sin n_{2}\left(t-t_{2}\right)+\text { const } .
$$

Using $e_{2}=1.673 \times 10^{-2}$ and $G M / c^{2} a_{2}=9.87 \times 10^{-9}$, the perturbation (3.9) corresponds to an along-track displacement

$$
a \delta_{1} \ell=-\left(\bar{\beta}-\frac{1}{4} \bar{\gamma}\right) \hat{a}^{-1 / 2} \sin \left(2 \pi \frac{t-t_{2}}{1 \mathrm{yr}}\right) \times 5.25 \times 10^{3} \mathrm{~cm} .
$$

The yearly modulation of $G_{\text {loc }}=\left(1-\eta G M / c^{2} D\right) G$ has other consequences which, in principle, enter satellite data through the motion of, e.g., Laser stations on the ground used to track a satellite. First, the radius of the Earth will undergo a corresponding yearly modulation. Using $\partial \ln R / \partial \ln G \simeq-0.1$ [22], the amplitude of this modulation is

$$
\begin{aligned}
\delta_{1} R & \simeq-0.1 R \frac{\delta G_{\mathrm{loc}}}{G_{\mathrm{loc}}} \simeq 0.1 R e_{2} \frac{4 G M}{c^{2} a_{2}}\left(\bar{\beta}-\frac{1}{4} \bar{\gamma}\right) \cos n_{2}\left(t-t_{2}\right) \\
& \simeq\left(\bar{\beta}-\frac{1}{4} \bar{\gamma}\right) \cos \left(2 \pi \frac{t-t_{2}}{1 \mathrm{yr}}\right) \times 4.21 \times 10^{-2} \mathrm{~cm}
\end{aligned}
$$

which is negligibly small. Second, this yearly breathing of the Earth will entail a corresponding modulation of its angular velocity, say $\Omega_{2}$. Using $\delta \Omega_{2} / \Omega_{2}=-\delta \mathcal{I}_{2} / \mathcal{I}_{2}$ and $\delta \mathcal{I}_{2} / \mathcal{I}_{2} \simeq-0.17 \delta G / G$ [23] where $\mathcal{I}_{2}$ denotes the Earth inertia moment, this leads, by integration, to a yearly modulation of the angle of rotation of the Earth and therefore a corresponding longitudinal displacement of ground stations:

$$
\begin{aligned}
R \delta_{1} \varphi & \simeq-0.17 R \frac{\Omega_{2}}{n_{2}} e_{2} \frac{4 G M}{c^{2} a_{2}}\left(\bar{\beta}-\frac{1}{4} \bar{\gamma}\right) \sin n_{2}\left(t-t_{2}\right) \\
& \simeq-\left(\bar{\beta}-\frac{1}{4} \bar{\gamma}\right) \sin \left(2 \pi \frac{t-t_{2}}{1 \mathrm{yr}}\right) \times 26.1 \mathrm{~cm} .
\end{aligned}
$$

Again, this effect is negligible compared to the orbital effect (3.10). [It would moreover probably be impossible to distinguish from other yearly modulations in the rotation of the Earth.]

It remains to study the orbital effects of the terms proportional to $G M / c^{2} D^{2}$ in the equations of motion. One checks that the short-period effects associated with $\mathbf{B}_{2}$, Eq. (2.7), are too small to be observationally relevant. One can get the secular effects of the orbital 
elements caused by $\mathbf{B}_{2}$ by averaging over one orbital period the time derivatives of the energy, the angular momentum and the Lagrange-Laplace (-Runge-Lenz) vector. One finds that $\langle d a / d t\rangle_{2}=0$ and

$$
\begin{aligned}
& \langle d \mathbf{e} / d t\rangle_{2}=\mathbf{f}_{2} \times \boldsymbol{\ell}, \\
& \langle d \boldsymbol{\ell} / d t\rangle_{2}=\mathbf{f}_{2} \times \mathbf{e},
\end{aligned}
$$

where $\mathbf{e}$ denotes the eccentricity vector of the orbit (i.e. a vector of magnitude $e$ directed toward the perigee), $\boldsymbol{\ell} \equiv \sqrt{1-e^{2}} \mathbf{c}$, with $\mathbf{c}$ a unit vector along the orbital angular momentum, and where the average "forcing term" caused by $\mathbf{B}_{2}$ is

$$
\begin{aligned}
\mathbf{f}_{2} & =k \frac{G M}{D^{2}} \mathbf{N}, \\
k & \equiv \frac{3}{2 n a}\left(\bar{\delta}_{1}-\bar{\delta}_{2}\right)-\frac{n a}{2 c^{2}}(4 \bar{\beta}-\bar{\gamma}) .
\end{aligned}
$$

In the general case of eccentric orbits, Eq. (3.13b) shows that $\mathbf{B}_{2}$ will produce variations of the inclination $I$ and the node $\Omega$ to the orbit of the satellite. Here, we shall concentrate on the more usual case where the eccentricity is small. Then, Eq. (3.13a) with $\boldsymbol{\ell} \simeq \mathbf{c}$ gives the contribution of $\mathbf{B}_{2}$ to the variation of $\mathbf{e}$. We must add the Newtonian effects of the quadrupole moment of the Earth $\left(J_{2}=1.08263 \times 10^{-3}\right)$. Let us introduce the basis $(\mathbf{a}, \mathbf{b})$ of the orbital plane where $\mathbf{a}$ is directed toward the ascending node and $\mathbf{a} \times \mathbf{b}=\mathbf{c}$. Because of the Newtonian effects of $J_{2}$ this basis is rotating around the Earth's polar axis with angular velocity $\dot{\Omega} \simeq-\frac{3}{2} n J_{2} \hat{a}^{-2} \cos I$ (see e.g. 可). Let $d^{\prime} / d t$ denote a time derivative in the rotating frame $(\mathbf{a}, \mathbf{b}, \mathbf{c})$. We get the following equation for the secular time evolution of the two independent components of $\mathbf{e}$ with respect to the vectors $(\mathbf{a}, \mathbf{b})$

$$
d^{\prime} \mathbf{e} / d t=\mathbf{c} \times\left[\dot{\omega}_{N} \mathbf{e}-\mathbf{f}_{\perp}(t)\right],
$$

where $\mathbf{f}_{\perp} \equiv\left(\mathbf{f}_{2} \cdot \mathbf{a}\right) \mathbf{a}+\left(\mathbf{f}_{2} \cdot \mathbf{b}\right) \mathbf{b}$ denotes the projection of $\mathbf{f}_{2}$ onto the orbital plane, and where

$$
\begin{aligned}
\dot{\omega}_{N} & =\frac{3}{4} \frac{n J_{2}}{\hat{a}^{2}}\left(4-5 \sin ^{2} I\right) \\
& \simeq\left(4-5 \sin ^{2} I\right) \hat{a}^{-7 / 2} \times 2 \pi /(0.20 \mathrm{yr})
\end{aligned}
$$

is the Newtonian perigee advance due to the Earth's quadrupole moment [5]. Eq. (3.15) constitutes an inhomogeneous linear differential equation in $\mathbf{e}=e \cos \omega \mathbf{a}+e \sin \omega \mathbf{b}$. The time dependence of the forcing term $\mathbf{f}_{\perp}(t)$ comes from a combination of the yearly variation of $\mathbf{f}_{2}$, Eq. (3.14a), with the rotation of the orbital plane with angular velocity $\dot{\Omega}$ around the Earth's polar axis. Following our recent work [5], we can easily solve for e $(t)$ by decomposing $\mathbf{f}_{\perp}$ in a sum of constant-norm vectors $\mathbf{f}_{\alpha}$ rotating with constant angular frequencies $\dot{\omega}_{\alpha}$ in the $(\mathbf{a}, \mathbf{b})$-plane: $\mathbf{f}_{\perp}=\Sigma_{\alpha} \mathbf{f}_{\alpha}$. Alternatively, one can work with complex numbers, $x \mathbf{a}+y \mathbf{b} \rightarrow$ $z \equiv x+i y$, and decompose $\mathbf{f}_{\perp} \cdot(\mathbf{a}+i \mathbf{b})$ into harmonic components $\propto \exp \left(i \dot{\omega}_{\alpha} t\right)$. Then the general solution of (3.15) has the form

$$
\mathbf{e}(t)=\mathbf{e}_{N}(t)+\sum_{\alpha} \mathbf{e}_{\alpha}(t),
$$


where

$$
\mathbf{e}_{\alpha} \equiv \frac{\mathbf{f}_{\alpha}}{\dot{\omega}_{N}-\dot{\omega}_{\alpha}}
$$

and where $\mathbf{e}_{N}(t)$ is a constant-norm vector rotating in the $(\mathbf{a}, \mathbf{b})$-plane with angular velocity $\dot{\omega}_{N}$ (homogeneous solution of (3.15)). In terms of complex numbers, $z=\mathbf{e} \cdot(\mathbf{a}+i \mathbf{b})=$ $e \exp i \omega$, this general solution reads

$$
\begin{array}{rl}
{[e(t) \exp i \omega(t)]_{2}=e_{N}} & \exp i\left(\dot{\omega}_{N} t+\sigma_{N}\right) \\
+k & G M\left[\frac{\left(1+\cos I_{2}\right)(1+\cos I)}{4 D^{2}} \exp +i\left(n_{2} t-\Omega\right)\right. \\
& +\frac{\left(1+\cos I_{2}\right)(1-\cos I)}{\dot{\omega}_{N}-\dot{\Omega}+n_{2}} \exp -i\left(n_{2} t-\Omega\right) \\
& +\frac{\left(1-\cos I_{2}\right)(1+\cos I)}{\dot{\omega}_{N}+\dot{\Omega}+n_{2}} \exp -i\left(n_{2} t+\Omega\right) \\
& +\frac{\left(1-\cos I_{2}\right)(1-\cos I)}{\dot{\omega}_{N}-\dot{\Omega}-n_{2}} \exp +i\left(n_{2} t+\Omega\right) \\
& +\frac{2 \sin I_{2} \sin I}{\dot{\omega}_{N}-n_{2}} \exp +i n_{2} t \\
& \left.+\frac{2 \sin I_{2} \sin I}{\dot{\omega}_{N}+n_{2}} \exp -i n_{2} t\right]
\end{array}
$$

where $I_{2}=23.45^{\circ}$ is the inclination of the apparent orbit of the Sun with respect to the Earth equatorial plane, and where we have neglected the eccentricity $e_{2}$ of the Earth's orbit.

We see clearly from Eq. (3.19) that the effects of $\mathbf{B}_{2}$ can be enhanced by small divisors if the semi-major axis and the inclination of the satellite orbit (on which both $\dot{\omega}_{N}$ and $\dot{\Omega}$ depend) are appropriately chosen. The locus of these resonances in the $(a, I)$ plane is the same as for the perturbations due to the orbital velocity of the Earth around the Sun in non-boost-invariant gravity theories. It will be found in Fig. 3 of [5]. Note that these resonances exist only if $a$ is smaller than $2.65 R$. Because of the smallness of $I_{2}$, the dominant contributions in the square brackets on the right-hand side of Eq. (3.19) are the first two ones corresponding to the frequencies $\pm\left(n_{2}-\dot{\Omega}\right)$ (see the solid lines in Fig. 3 of [5]). However, the price to pay to take advantage of these resonances is to dispose of a long time of observation. In this work we have in mind using tracking data from missions dedicated to other purposes (e.g. GPB or STEP) which may last only for a year or so. Then, the best situation is when $\dot{\omega}_{N} \pm\left(\dot{\Omega}-n_{2}\right)$ is of order $2 \pi / 1$ yr. In such a case, we see from Eq. (3.19) that $\mathbf{B}_{2}$ will cause spatial displacements with periods $\sim 1$ yr and with amplitudes

$$
\begin{aligned}
a \delta_{2} e \sim a k \frac{G M}{2 D^{2}} \frac{1 \pm \cos I}{\dot{\omega}_{N} \pm\left(\dot{\Omega}-n_{2}\right)} \\
\sim\left[1.80 \times 10^{9} \hat{a}^{3 / 2}\left(\bar{\delta}_{1}-\bar{\delta}_{2}\right)-1.67 \hat{a}^{1 / 2}\left(\bar{\beta}-\frac{1}{4} \bar{\gamma}\right)\right] \\
\quad \times(1 \pm \cos I) \frac{2 \pi / 1 \mathrm{yr}}{\dot{\omega}_{N} \pm\left(\dot{\Omega}-n_{2}\right)} \mathrm{cm}
\end{aligned}
$$


where $\bar{\delta}_{1}-\bar{\delta}_{2} \simeq \widehat{\delta}_{1}-\widehat{\delta}_{2}+1.86 \times 10^{-9}(\bar{\beta}-\bar{\gamma} / 4)$, cf. Eq. (1.3). For low Earth satellites $(\hat{a} \sim 1)$ this is much smaller than the effects of $\mathbf{B}_{0}$ and $\mathbf{B}_{1}$ discussed above, and will therefore not contribute much to the measurability of $\bar{\beta}, \bar{\gamma}$ and $\bar{\delta}$. By comparing however the negative power law dependences upon $\hat{a}$ of Eqs. (3.2) and (3.10) with the corresponding positive power dependences in Eq. (3.20) we see in retrospect why the most sensitive non-Einsteinian test in the motion of the Moon $(\hat{a} \simeq 60)$ can be the violation of the equivalence principle (term $\propto \bar{\delta}_{1}-\bar{\delta}_{2}$ in $k$ [12,9]).

Finally, let us consider for completeness the orbital effects of a slow time variation of $G$ and/or the masses. Going back to the level of the original Lagrangian (1.1) we must consider that the mass of the satellite $m_{s}$, the mass of the Earth $m$ and $G$ can vary in the Newtonian-approximation Lagrangian for the geocentric motion:

$$
L_{N}=\frac{1}{2} m_{s}(t)\left(\frac{d \mathbf{r}}{d t}\right)^{2}+\frac{G(t) m_{s}(t) m(t)}{r} .
$$

We can solve for the dynamics of $L_{N}$ by a simple generalization of the method used above for the yearly variation of $G_{\text {loc }}$.

Indeed, let us consider more generally a perturbed Lagrangian of the type $L=L_{0}+L_{1}$ with $L_{0}=\frac{1}{2} \mathbf{v}^{2}+G m / r$ and $L_{1}=\frac{1}{2} a(t) \mathbf{v}^{2}+b(t) G m / r$ where $G m$ is constant and where $a(t)$, $b(t) \ll 1$. Let us introduce the following new space and time variables

$$
\begin{aligned}
\mathbf{r}^{\prime} & =(1+a+b) \mathbf{r} \\
t^{\prime} & =t+\int d t(a+2 b) .
\end{aligned}
$$

By using Eqs. (3.4) and (3.5) we find that

$$
L^{\prime}=L_{0}+\frac{1}{2} \mathbf{r}^{2} \frac{d^{2}}{d t^{2}}(a+b)+\frac{d}{d t} Q^{\prime}
$$

We can apply this result to the Lagrangian (3.21) decomposed as $L_{N}=m_{s}\left[L_{0}+L_{1}\right]$ by writing $m_{s}(t)=m_{s}\left(1+t \dot{m}_{s} / m_{s}\right), m(t)=m(1+t \dot{m} / m), G(t)=G(1+t \dot{G} / G)$ (where $m_{s}, m$ and $G$ denote some constants). [Here, one is making the assumption that the characteristic time scale $T$ of variation of $G(t), m_{s}(t), m(t)$ is very large so that one can work to first order in $T^{-1}$.] This yields $a=t \dot{m}_{s} / m_{s}$ and $b=t\left(\dot{G} / G+\dot{m} / m+\dot{m}_{s} / m_{s}\right)$, and therefore $\left(d^{2} / d t^{2}\right)(a+b)=0$ in Eq. (3.23). Finally, we get that the motion in terms of the variables

$$
\begin{aligned}
\mathbf{r}^{\prime} & =\left[1+t\left(\frac{\dot{G}}{G}+\frac{\dot{m}}{m}+2 \frac{\dot{m}_{s}}{m_{s}}\right)\right] \mathbf{r} \\
t^{\prime} & =t+\frac{1}{2} t^{2}\left(2 \frac{\dot{G}}{G}+2 \frac{\dot{m}}{m}+3 \frac{\dot{m}_{s}}{m_{s}}\right),
\end{aligned}
$$

is described by the unperturbed Lagrangian $L_{0}$.

Knowing already that $|\dot{G} / G|<10^{-11} \mathrm{yr}^{-1}$ and $|\dot{m} / m|<10^{-13} \mathrm{yr}^{-1}$ we see that the effects (3.24a) on the size of the orbit is too small to be observable in satellite data. On the other hand the effect (3.24b) leads to a mean anomaly perturbation 


$$
\ell=n t^{\prime}+\mathrm{const}=n t+\frac{1}{2} n t^{2}\left(2 \frac{\dot{G}}{G}+2 \frac{\dot{m}}{m}+3 \frac{\dot{m}_{s}}{m_{s}}\right)
$$

corresponding to an along-track displacement?

$$
\begin{aligned}
a \delta \ell & =n a t^{2}\left(\frac{\dot{G}}{G}+\frac{\dot{m}}{m}+\frac{3}{2} \frac{\dot{m}_{s}}{m_{s}}\right) \\
& \simeq \hat{a}^{-1 / 2}\left(\frac{\dot{G}}{G}+\frac{\dot{m}}{m}+\frac{3}{2} \frac{\dot{m}_{s}}{m_{s}}\right) t^{2} \times 2.50 \times 10^{13} \mathrm{~cm} \mathrm{yr}^{-1}
\end{aligned}
$$

which suggests that the level $\dot{G} / G \sim 10^{-13} \mathrm{yr}^{-1}$ might be reachable by satellite data [assuming that the ordinary sources of variation of the mass of the Earth are smaller, or, at least, measurable with better precision].

\section{CONCLUSIONS}

We have reexamined the non-Einsteinian effects in the orbital motion of low-orbit artificial Earth satellites. We worked within the most conservative framework for alternative gravity theories, i.e. the assumption that gravity contains, besides the usual Einsteinian tensor interaction, a scalar contribution. The post-Newtonian limit of generic tensor-scalar gravity theories exhibit three types of non-Einsteinian effects: (i) deviations from the general relativistic post-Newtonian effects, parametrized by two (Eddington) parameters $\bar{\beta} \equiv \beta-1$, and $\bar{\gamma} \equiv \gamma-1$, (ii) combined violations of the weak and strong equivalence principles, parametrized by body-dependent parameters $\bar{\delta}_{A} \equiv \widehat{\delta}_{A}+(4 \bar{\beta}-\bar{\gamma}) E_{A}^{\text {grav }} / m_{A} c^{2}$; and (iii) possible slow time variabilities of Newtonian parameters, measured by $\dot{G} / G$ and $\dot{m}_{A} / m_{A}$.

Let us recall that the Lunar Laser Ranging (LLR) experiment has, as predicted [12,9], proven to be a superb tool for measuring $\bar{\delta}_{\text {Moon }}-\bar{\delta}_{\text {Earth }}=(-2.7 \pm 6.2) \times 10^{-13}$, i.e. for testing the validity of the effacement of the internal structure of the bodies in their translational equations of motion. The present paper indicates that the centimeter-level tracking of loworbit drag-free satellites is a promising tool for measuring at the $10^{-4}$ level two independent combinations of $\bar{\beta}$ and $\bar{\gamma}\left[\bar{\beta}-2 \bar{\gamma}\right.$ Eq. (3.1) and $\bar{\beta}-\frac{1}{4} \bar{\gamma}$, Eq. (3.10)] and for probing the time variability of $G$ (and the masses) at the $10^{-13} \mathrm{yr}^{-1}$ level, Eq. (3.26). It is interesting to notice that the satellite measurement of $\eta \equiv 4 \bar{\beta}-\bar{\gamma}$ is complementary to the LLR measurement of $\bar{\delta}_{1}-\bar{\delta}_{2}$ in two ways: (i) conceptually it is a test of the effacement of the external universe in the equations of motion of a local system, and (ii) technically it gives directly access to the parameter $\eta$ which is "contaminated" by possible weak-equivalence-principle violations in the LLR observable $\bar{\delta}_{1}-\bar{\delta}_{2}=\widehat{\delta}_{1}-\widehat{\delta}_{2}+\eta \times 4.45 \times 10^{-10}$ [15].

\footnotetext{
${ }^{9}$ The result $(3.26)$ assumes that one has experimentally access to the basic dynamical time $t$. Taking into account that time is measured by atomic clocks based on, e.g., a Bohr frequency $\propto m_{e} \alpha^{2}$ adds the terms $-\frac{1}{2} \dot{m}_{e} / m_{e}-\dot{\alpha} / \alpha$ in the parenthesis on the right-hand side of Eq. (3.26).
} 
A crucial difficulty in reaching the precision levels suggested by our analysis will be to protect the motion of the satellite from non-gravitational forces ${ }^{\mathrm{D}} \mathrm{t}$. The most promising orbital effects, Eqs. (3.1), (3.10) and (3.26), come from the build up of small effects over observation times of the order of one year. The drag-free system of the satellite must therefore be effective for correspondingly ultra low frequencies. It may well be that the active drag compensation systems of the type planned for the GPB satellite will fall short of providing the needed protection. The best system would probably be a passive drag-free system where the satellite floats freely within a large shell.

If it appears impossible to use the orbital data of drag-free satellites conceived for other missions [like GPB or the Satellite Test of the Equivalence Principle (STEP)], it may be worth thinking about a dedicated mission [say the Satellite Test of the Effacement property (STEFF)] consisting of an optimized passive drag-free satellite: a small ball freely floating within a large shell which follows optically the motion of the ball and which carries some thrusters and GPS or DORIS receivers and/or laser corner cubes. The orbital data of such a mission would be of value both for geodesy and as tests of relativistic gravity.

Other difficulties concern the knowledge of the multipole moments of the Earth which limit other proposals (see [7]). For instance, the present uncertainty in the Earth quadrupole $J_{2}, \delta J_{2} / J_{2} \sim 6 \times 10^{-7}$ [24] contributes an uncertainty in the predicted (Newtonian) value of the perigee advance which corresponds to the uninteresting level $\delta(\bar{\beta}-2 \bar{\gamma}) \sim 1$. This is however not a serious difficulty as the high-precision satellite data that we wish to use to get (as by products) measurements of $\bar{\beta}$ and $\bar{\gamma}$, will be primarily used anyway to refine the measurement of the Earth gravity field with a correspondingly high precision (see e.g. [18]).

One might think that the type of orbital tests we have been discussing will soon loose their interest as other planned experiments aim at reaching higher precisions in measuring possible deviations from General Relativity. [We have in mind, in particular, Gravity Probe B (which should measure $\bar{\gamma}$ at the $3 \times 10^{-5}$ level [25]) and the Solar Orbit Relativity Test (SORT) [26] which aims at the $10^{-7}$ level for $\bar{\gamma}$.] As these tests will not measure $\bar{\beta}$, we see on the contrary that orbital tests may provide our best handle on the parameter $\bar{\beta}$. Assuming the knowledge of $\bar{\gamma}$, the present analysis shows that orbital data will contain two independent signals, Eq. (3.1) and (3.10), for measuring $\bar{\beta}$ at the $10^{-4}$ level. One can note that while the perigee advance of Eq. (3.1) is difficult to measure in the usual case of loweccentricity orbits, the yearly modulation (3.10) is essentially independent of the magnitude of $e$. It remains however to see to what extent it will possible to distinguish it from the other yearly (gravitational) perturbations in the orbit. [Note that the phase of the signal (3.10) is predicted and that the period is the anomalistic year (perihelion to perihelion).] Let us also remark that, contrary to many of the effects discussed in [5], the magnitudes of the leading perturbations we have discussed, Eqs. (3.1), (3.10) and (3.26), are independent

\footnotetext{
${ }^{10}$ One notes in particular that solar radiation pressure effects mimic exactly the yearly variation of $G_{\text {loc }}$ but are several orders of magnitude larger.

${ }^{11}$ Let us note in passing that the scenario of Ref. [4] indicates that the ratio $-\bar{\beta} / \bar{\gamma}=\beta_{3} / 4$ might be of order 10 (if $\kappa \sim 1$ ).
} 
of the value of the inclination of the orbit. A strong dependence upon $I$ appeared only in the subdominant effects caused by $\mathbf{B}_{2}$, Eq. (3.19).

Finally, let us stress that the value of the approximate analytical solutions for nonEinsteinian perturbations that we have given above is mainly indicative of the results one can expect. In practice, we advise to resort to direct numerical integration of the equations of motion (2.2). For $\mathbf{A}_{\mathrm{GR}}$ one should use the full general relativistic post-Newtonian equations of motion [10], to which one can add linearly the effect of $\mathbf{B}$. The accuracy with which we have given B in Eqs. (2.5)-(2.7) should be amply sufficient. [If one wishes one can also consider the non-Einsteinian effect associated with the spin angular momentum of the Earth $\mathbf{S}_{2}$, i.e. add the term $\mathbf{B}_{S}$, Eq. (2.9), to $\mathbf{B}$.] 


\section{REFERENCES}

[1] C.M. Will, Theory and Experiment in Gravitational Physics, (Cambridge University Press, Cambridge, 1992). (second edition).

[2] T. Damour, in Gravitation and Quantizations, Proceedings of the LVIIth Les Houches Summer School, July 1992, edited by B. Julia and J. Zinn-Justin (North-Holland, Amsterdam, 1994).

[3] T. Damour and K. Nordtvedt, Phys. Rev. Lett. 70, 2217 (1993); Phys. Rev. D 48, 3436 (1993).

[4] T. Damour and A.M. Polyakov, submitted to Nucl. Phys. B (1994).

[5] T. Damour and G. Esposito-Farèse, Phys. Rev. D 49, 1693 (1994).

[6] M. Soffel, Relativity in Astrometry, Celestial Mechanics and Geodesy (Springer-Verlag, Berlin, 1989).

[7] I. Ciufolini, Int. J. Mod. Phys. A 4, 3083 (1989).

[8] V.A. Brumberg, Essential Relativistic Celestial Mechanics (Adam Hilger, Bristol, 1991).

[9] K. Nordtvedt, Phys. Rev. D 7, 2347 (1973).

[10] T. Damour, M. Soffel and C. Xu, Phys. Rev. D 43, 3272 (1991); 45, 1017 (1992); 47, 3124 (1993); and 49, 618 (1994).

[11] T. Damour and G. Esposito-Farèse, Class. Quant. Grav. 9, 2093 (1992).

[12] K. Nordtvedt, Phys. Rev. 169, 1014; 169, 1017; 170, 1186 (1968).

[13] R.D. Reasenberg et al., Astrophys. J. 234, L219 (1979).

[14] I.I. Shapiro, in General Relativity and Gravitation, 1989, ed. N. Ashby, D.F. Bartlett and W. Wyss (Cambridge University Press, Cambridge, 1990), pp. 313-330.

[15] J.O. Dickey et al., Science, 1994, in press.

[16] P. Sisterna and H. Vucetich, Phys. Rev. D 41, 1034 (1990).

[17] Y. Su et al., Phys. Rev. D, 1994, submitted.

[18] M. Tapley, Adv. Space Research 13, (7)77 (1993).

[19] T. Damour, in 300 Years of Gravitation, ed. S.W. Hawking and W. Israel (Cambridge University Press, Cambridge, 1987) pp. 128-198.

[20] T. Damour and N. Deruelle, Ann. Inst. H. Poincaré, 43, 107 (1985).

[21] T. Damour and G. Schäfer, Gen. Rel. Grav. 17, 879 (1985).

[22] C.T. Murphy and R.H. Dicke, Proc. Amer. Phil. Soc. 108, 224 (1964)

[23] R.A. Lyttleton and J.P. Fitch, Astrophys. J. 221, 412 (1978)

[24] A. Milani, A.M. Nobili and P. Farinella, Non-gravitational Perturbations and Satellite Geodesy (Adam Hilger, Bristol, 1987).

[25] S. Buchmann and C.W.F. Everitt, contribution to the Proceedings of the XIVth Moriond Workshop on "Particle Astrophysics, Atomic Physics and Gravitation" (Editions Frontières, Gif sur Yvette, 1994), in press.

[26] C. Veillet et al., proposal in response to the European Space Agency call for mission concepts for the follow-up to Horizon 2000, October 1993. 